\title{
Foreword
}

\section{Sudden Cardiac Death: Back to the Future}

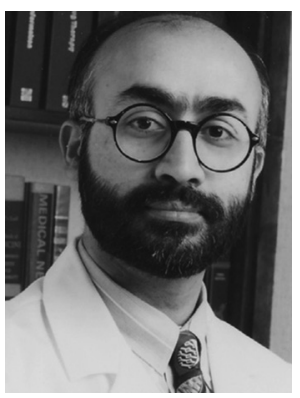

Ranjan K. Thakur, MD, MPH, MBA, FHRS

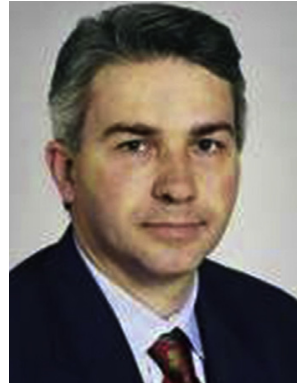

Andrea Natale, MD, FACC, FHRS

Consulting Editors

We are pleased to introduce this issue of Cardiac Electrophysiology Clinics on sudden cardiac death (SCD). In the 1960s and 1970s, a focus on arrhythmia mechanisms, ventricular arrhythmias, and SCD ushered in the field of cardiac electrophysiology and paved the way for adoption of the implantable cardioverter defibrillator. Befittingly, when Cardiac Electrophysiology Clinics was launched in December 2009, the topic chosen for a comprehensive review was SCD. Having covered myriad topics in electrophysiology over the past 8 years, we return once again, on the eighth anniversary of Cardiac Electrophysiology Clinics, to revisit the issue of SCD.

Despite decreasing cardiovascular mortality, including SCD mortality over the last few decades, it still remains a major public health issue, and there is much room for improvement in identifying those at risk and preventing SCD. Also, while per capita cardiovascular mortality is decreasing in the developed world, it may be increasing in much of the developing world, and therefore, it remains a prominent issue on a global scale. This issue of Cardiac Electrophysiology Clinics focuses on contemporary approaches and thoughts relevant to SCD.

We thank Drs Shenasa, Estes, and Tomaselli for editing this issue of Cardiac Electrophysiology
Clinics. They have invited expert contributors to summarize the current thinking on mechanisms of SCD, SCD in athletes and adolescents, identification of at-risk populations, and risk stratification, prevention, emergency management, and prophylaxis of SCD, to name a few topics covered in this issue. Electrophysiologists, cardiologists, and fellows in training will find these comprehensive review articles useful to bring them up to speed on contemporary ideas and controversies.

Ranjan K. Thakur, MD, MPH, MBA, FHRS Sparrow Thoracic and Cardiovascular Institute Michigan State University

1200 East Michigan Avenue, Suite 580 Lansing, MI 48912, USA

Andrea Natale, MD, FACC, FHRS Texas Cardiac Arrhythmia Institute

Center for Atrial Fibrillation at

St David's Medical Center 1015 East 32nd Street, Suite 516

Austin, TX 78705, USA

E-mail addresses:

thakur@msu.edu (R.K. Thakur) andrea.natale@stdavids.com (A. Natale) 